\title{
Inter-laboratory comparison on the calibration of measurements photometric and radiometric sensors
}

\author{
K. Berk Sönmez ${ }^{1, *}$, T. Oytun Kılınç ${ }^{1}$ İ. Ahmet Yüksel ${ }^{1}$, Sinem Ön Aktan ${ }^{1}$ \\ ${ }^{1}$ Department of Laboratories and Calibration Management, Roketsan Rocket Industry and Trade Inc., \\ 06780 Ankara, Turkey
}

\begin{abstract}
The competences of the test and calibration laboratories are provided by two complementary methods. The first of these methods is the on-site audit carried out by the administrative and technical committee established by TURKAK (Turkish Accreditation Institution) according to the requirements of TS EN ISO / IEC 17025 [1]. The other technique is interlaboratory comparison and proficiency testing to evaluate laboratory performance and ensure the quality of results. Investing in the right equipment, training personnel, defining methods, documenting, calculating uncertainty, even performing internal verifications do not guarantee reliability or accuracy. It cannot answer the question of whether we can produce the same results as the same testers in the world. It is necessary to prove that the laboratory can actually produce accurate results externally by going through comparison tests with other national/international laboratories.
\end{abstract}

\section{Introduction}

Inter-laboratory comparison (ILC) means organization, implementation and evaluation of the results of measurement and testing of the same or similar test items/samples carried out by two or more than two laboratories in conformity with pre-determined conditions. The inter laboratory comparison consists in testing the same samples by different laboratories and in comparing the results. The principle of this inter-laboratory comparison was to circulate three parameters from one laboratory to another in order to compare the calibration results. These are including systematic error, standard deviation and calibration uncertainty. The connection of each parameter included in the ILC tests is given in figure 1.

*Corresponding Author : kemalberksonmez@gmail.com 


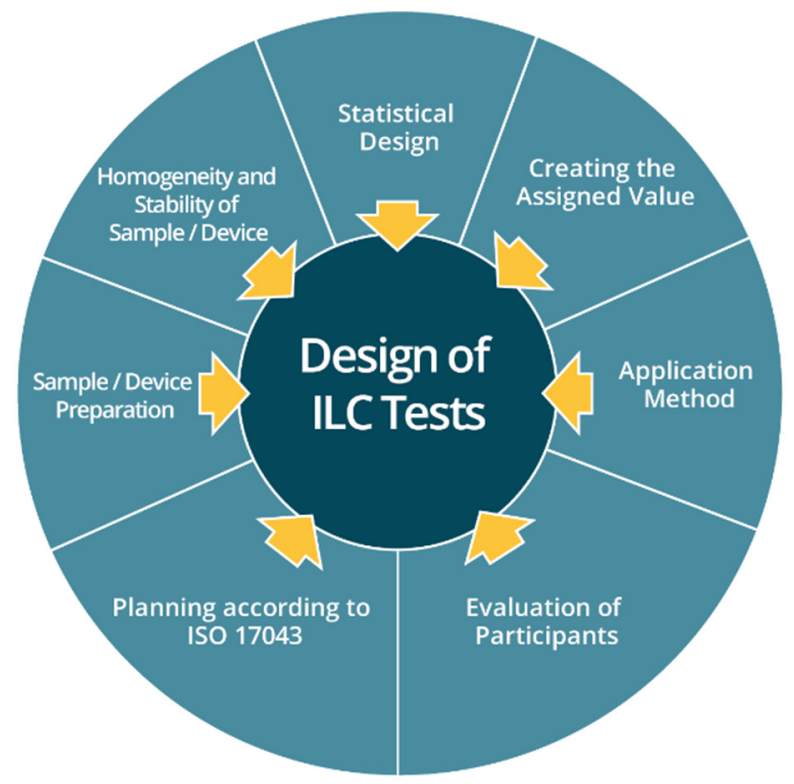

Fig. 1. Design of Interlaboratory Comparison Tests Diagram

In this study, the inter-laboratory comparison test organized jointly by the Roketsan's optical calibration department and the related of a Turkish Aviation Company calibration department was successfully completed. The main objective of participation in these comparisons is the evaluation of the suitability of the laboratories to perform measurements according to a given normative document such as ISO/IEC 17025. In order to achieve this test, the comparison laboratory was selected with a technical department, which is an accredited institution in a Turkish Aviation Company. Participation in measurement comparison is one of the main mechanisms used by calibration laboratories to provide evidence of their technical competence to their customers and stakeholders. Successful participation in comparisons is also one of the elements by which non-accredited laboratories can demonstrate their competence at accreditation bodies (ABs).

Different methods are available to exploit the results of participants and are described in standards. Each participant used its own calibration procedures and issued calibration certificates according to the ISO17025 standard. You can see the method, material and performance operations in Figure 2.

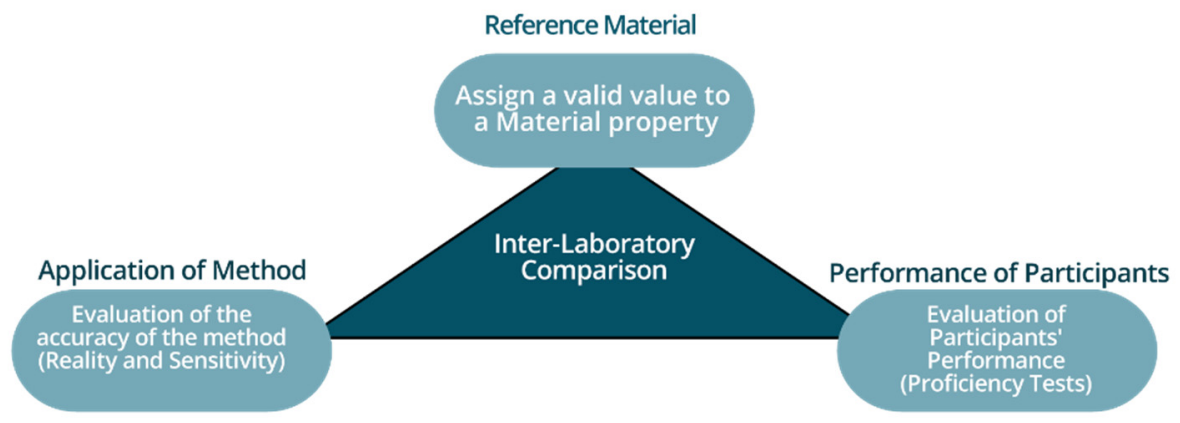

Fig. 2. Reference Material, Application of Method and Performance of Participants 
The recommended calibration standard is the international commission on illumination methods. These are;

$\checkmark$ CIE198 Determination of measurement uncertainties in photometry,

$\checkmark$ CIE220 Characterization and calibration methods of UV radiometers,

$\checkmark$ CIE202 Spectral responsivity measurement of detectors, Radiometers and Photometers.

According to TS EN ISO / IEC 17025 standard, accreditation is not a requirement for laboratories, but it is essential to participate in pre-audit benchmark tests and produce successful results. Participation in comparison tests at least once in 48 months has to be repeated so that the performance of the laboratory is continuously monitored. The frequency of participation in proficiency tests varies according to the number of tests, staff turnover rate, experience of the technical team, importance of final use and traceability source [2]. Briefly, the comparison of the capabilities of the two laboratories in the relevant measurements and the comparison results was reported by specifying the uncertainties.

Accurate, reliable testing and measurements are essential at every moment of our lives and are becoming more critical in the defense industry. According to all quality documents and ISO 10012 "Measurement Management Systems - Conditions for Measuring Processes and Measuring Instruments" document [3], traceability of all measuring and test devices directly affecting product quality during the life of the product should be ensured according to national / international standards. In addition, in order to make the right decision about the processes, it is necessary to produce the right test results, to participate in the comparison tests for the product quality and the right production at the first time. The important information belonging to ILC were shared in the prism steps (see Fig. 3.).

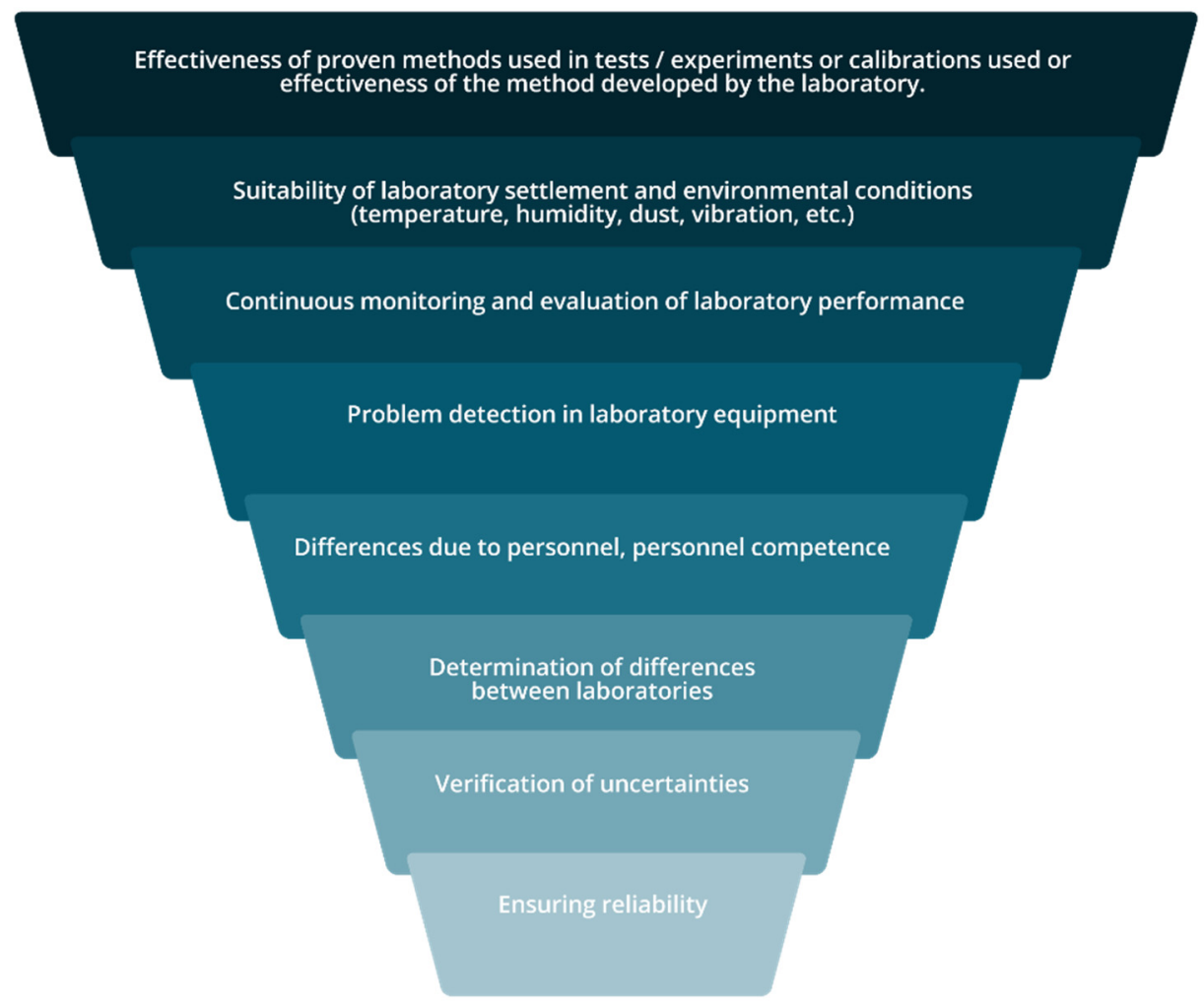

Fig. 3. Important information of ILC Tests 
Interlaboratory comparison (ILC) is the arrangement and planning of two or more laboratories with similar or similar substance ongoing tests or measurements according to predefined conditions [4].

ILC tests are conducted to confirm the accuracy of any method and sometimes to make a precise and highly accurate assignment of the value of a material.

As a result of ILC Tests, the performances of the laboratories can be evaluated with many different statistical approaches as described in ISO 13528 Standard [6]. The most commonly used method in evaluations is calculated by $\mathrm{E}_{\mathrm{n}}$ number. In order to use this method, participants need to master the uncertainty calculations.

\section{Methods}

In this study, we aim to compare the first one is related to photometric area $(380 \mathrm{~nm}-780$ $\mathrm{nm}$ ) for luminance measurement. The second one is related to radiometric area for UV measurement with UV-A region $(315 \mathrm{~nm}-380 \mathrm{~nm})$.

Photometry is the subject of light measurements between the wavelengths of $380 \mathrm{~nm}$ and $780 \mathrm{~nm}$, which can be detected by the human eye between the infrared and ultraviolet regions of the electromagnetic spectrum. Photometry is the measurement of electromagnetic radiation weighted by the human eye's response. This response changes with wavelength, and to an extent, from person to person. Internationally agreed standard observer functions are therefore used.

In general, photometric measurements in daily life use photometric devices such as Lux meter or Light Meter with a perception similar to direct human eye sensitivity. Lux meters consist of a white light diffuser on the outermost light, a green transparent optical filter that creates human eye sensitivity, and a silicon photodetector connected to the indicator electrical amplifier unit as a sensor. Photometric measurement refers to light related and laboratory measurements. The expression of the results of these measurements is also used in photometric measurements.

The luminance measurements are made to determine the magnitude of luminous flux from an object to a direction, for example, to our eye. Since the only detectable photometric size is luminance, these measurements are more important than expected. The object to be measured for luminance may be the primary light source, that is, a light generator that converts another kind of energy into light, or the secondary light source, that is, an object that sends light around it by reflecting or passing light onto it. The luminance of the secondary light sources is determined by the level of illumination on the object and the reflection factor of that surface or the transmission multiplier of that object. Luminance measurements are also used to determine the reflection and transmission factors of objects.

The candela is the luminous intensity, in a given direction, of a source, that emits monochromatic radiation of frequency $540 \times 10^{12}$ hertz and that has a radiant intensity in that direction of $1 / 683$ watts per steradian. [7]

\section{Measurements and analysis}

Illuminance is a measurement of radiant energy on a surface, weighted by the human eye response. Illuminance is quantified in units of lux or foot-candles. Lux is luminous flux incident on a surface in units of lumens per square meter and foot-candles is luminous flux incident on a surface in units of lumens per square foot. There are 10.7639 square feet in a square meter, so lux can be converted to foot-candles by dividing by 10.7639 . Sensors that measure illuminance are referred to by many names, including light sensors, photometric radiometers, optic sensors, and lux sensors. 
Light intensity I (cd) means the luminous flux emitted from a point in a given direction at a space angle (steradian). When we take 1 lumen $=1$ candela $\mathrm{x} 1$ steradian, the luminous flux at a steradian angle also gives us the light intensity. In addition, when explaining the Luminous Flux calculation equations, we expressed; $d A=d w \times r^{2}$. In this equation, $d w$ is a differential solid angle. And also, I; candela (cd) means the intensity of light. The illumination of ideal spot-type light sources will technically decrease in proportion to the inverse of the distance as you move away from the source, in accordance with the rule; $1 / \mathrm{r}^{2}$ technic. For this reason, it is important how far away from the light source the measurements are taken.

All measurements were taken with different brand of photometric and radiometric sensors. Photometric sensors measurement results were taken between 50 Lux and 200 Lux, radiometric sensor measurement results were taken between $2000 \mu \mathrm{w} / \mathrm{cm}^{2}$ and 4000 $\mu \mathrm{w} / \mathrm{cm}^{2}$. All the measurements conditions were $23.2 \pm 1{ }^{\circ} \mathrm{C}$ and $38.4 \% \mathrm{RH}$ (see table 1.)

Table 1. Conditions Table

\begin{tabular}{|c|c|}
\hline \multicolumn{2}{|c|}{ Conditions } \\
\hline Temperature $\left({ }^{\circ} \mathrm{C}\right)$ & $23.2 \pm 1$ \\
\hline Humidity $(\%)$ & 38.4 \\
\hline
\end{tabular}

In this study, ten measurements were taken in both photometric sensor and radiometric sensors with same conditions. The average values of the measurements were calculated and shown in two different tables (see table 2. and table 3.).

Table 2. Number of Photometric Measurements Table

\begin{tabular}{|c|c|c|c|c|c|c|}
\hline $\begin{array}{c}\text { Number of } \\
\text { Photometric } \\
\text { Measurements }\end{array}$ & \multicolumn{2}{|c|}{$\mathbf{5 0}$ (Lux) } & \multicolumn{2}{|c|}{$\mathbf{1 0 0}$ (Lux) } & \multicolumn{2}{|c|}{$\mathbf{2 0 0}$ (Lux) } \\
\cline { 2 - 7 } & 49.54 & 49.12 & 102.33 & 101.86 & 205.12 & 203.79 \\
\hline 1 & 49.56 & 49.11 & 102.31 & 101.92 & 205.08 & 203.73 \\
\hline 2 & 49.54 & 49.13 & 102.33 & 101.86 & 205.06 & 203.79 \\
\hline 3 & 49.55 & 49.13 & 102.34 & 101.74 & 205.06 & 203.85 \\
\hline 4 & 49.55 & 49.14 & 102.33 & 101.8 & 205.09 & 203.6 \\
\hline 5 & 49.53 & 49.12 & 102.33 & 101.8 & 205.08 & 203.73 \\
\hline 6 & 49.55 & 49.13 & 102.32 & 101.86 & 205.06 & 203.92 \\
\hline 7 & 49.56 & 49.12 & 102.33 & 101.8 & 205.09 & 203.79 \\
\hline 8 & 49.57 & 49.11 & 102.36 & 101.74 & 205.08 & 203.73 \\
\hline 9 & 49.53 & 49.12 & 102.34 & 101.67 & 205.05 & 203.73 \\
\hline 10 & 49.55 & 49.12 & 102.33 & 101.81 & 205.08 & 203.77 \\
\hline$X_{\text {average }}$ & 0.127263746 & 0.045780769 & 0.056832192 \\
\hline$E_{n}$ & & & & & & \\
\hline
\end{tabular}


Table 3. Number of Radiometric Measurements Table

\begin{tabular}{|c|c|c|c|c|c|c|}
\hline \multirow{2}{*}{$\begin{array}{c}\text { Number of } \\
\text { Radiometric } \\
\text { Measurements }\end{array}$} & B & $\mathbf{A}$ & B & $\mathbf{A}$ & B & $\mathbf{A}$ \\
\hline & \multicolumn{2}{|c|}{$2000\left(\mu \mathrm{w} / \mathrm{cm}^{2}\right)$} & \multicolumn{2}{|c|}{$3000\left(\mu \mathrm{w} / \mathrm{cm}^{2}\right)$} & \multicolumn{2}{|c|}{$4000\left(\mu w / \mathrm{cm}^{2}\right)$} \\
\hline 1 & 2183,5 & 2172,2 & 3057,2 & 3036,1 & 4859,4 & 4852,2 \\
\hline 2 & 2180,7 & 2168,3 & 3055,4 & 3035,7 & 4861,2 & 4749,6 \\
\hline 3 & 2179,9 & 2169,4 & 3051,2 & 3033,4 & 4864,5 & 4850,1 \\
\hline 4 & 2181,2 & 2170,6 & 3049,9 & 3032,8 & 4869,6 & 4856,5 \\
\hline 5 & 2189,6 & 2173,1 & 3052,3 & 3036,9 & 4864,1 & 4748,4 \\
\hline 6 & 2181,6 & 2169,7 & 3054,2 & 3035,4 & 4862,3 & 4854,3 \\
\hline 7 & 2188,3 & 2171,5 & 3051,6 & 3031,2 & 4864,2 & 4853,5 \\
\hline 8 & 2184,5 & 2170,6 & 3055,1 & 3040 & 4862,3 & 4852,1 \\
\hline 9 & 2183,4 & 2169,5 & 3052,9 & 3035,8 & 4866,9 & 4856,7 \\
\hline 10 & 2185,4 & 2174,6 & 3055,4 & 3037,8 & 4867,8 & 4853,1 \\
\hline $\mathrm{X}_{\text {average }}$ & 221,75 & 221,17 & 469,22 & 467,37 & 685,11 & 681,62 \\
\hline$E_{n}$ & \multicolumn{2}{|c|}{0,128113855} & \multicolumn{2}{|c|}{0,176820289} & \multicolumn{2}{|c|}{0,129658652} \\
\hline
\end{tabular}

Abbreviations for table 2 and table 3;

$\mathrm{B}=$ Turkish aviation company who serves worldwide

$\mathrm{A}=$ Roketsan Rocket Industry and Trade Inc.

Table 4. En Formula Table

\begin{tabular}{|l|}
\hline$X_{L a b}:$ Measurement Value of the Participating Laboratory \\
\hline$X_{R e f}:$ Measurement Value of the Reference Laboratory \\
\hline$U_{L a b}:$ Measurement Uncertainty of the Participating Laboratory \\
\hline$U_{R e f}:$ Measurement Uncertainty of the Reference Laboratory \\
\hline
\end{tabular}

$$
E_{n}=\frac{X_{A-X_{B}}}{\sqrt{U_{A}^{2}+U_{B}^{2}}}
$$

Table 5. Uncertainty Table

\begin{tabular}{|c|c|}
\hline \multicolumn{2}{|c|}{ Uncertainty (\%) } \\
\hline B & 0.82 \\
\hline A & 1.05 \\
\hline
\end{tabular}

As a result of ILC Tests;

$\left|E_{n}\right| \leq 1$ Participants who score 1 is sufficient.

$\left|E_{n}\right|>1$ Showed insufficient performance and action is expected from the laboratory to improve the result. 


\subsection{Uncertainties approaches}

The following model function is given equation 2 .

$$
E=\left(E-E_{0}\right)\left(\frac{T_{d}}{2856}\right)^{M}\left(1-\delta_{\text {str. }}\right)+\delta_{\text {hc. }}+\delta_{\text {res. }}+\delta_{\text {ref }}
$$

$\mathrm{E}_{0} \quad$ : Uncertainty from the coefficient of measurements in the dark

E : Uncertainty from the reproducibility of measurements

M : Coefficient of the light source color temperature

$\delta_{\text {ref }}:$ Uncertainty from the reference sensor's certificate

$\delta_{h c .}:$ Uncertainty from the high caliper

$\delta_{\text {str. }}$ : Uncertainty from leakage light measurements

$\delta_{\text {res. }}$ : Uncertainty from the resolution of radiometer devices

$T_{d}$ : Uncertainty from the color temperature of the light source used

Table 6. Uncertainty Budget Table

\begin{tabular}{|l|c|c|c|}
\hline Definition & Symbol & Distribution & Factor \\
\hline $\begin{array}{l}\text { Photometer Header } \\
\text { Certificate }\end{array}$ & $\delta_{\text {ref. }}$ & Normal & 2 \\
\hline Radiometer Resolution & $\delta_{\text {res. }}$ & Rectangle & 1,73 \\
\hline $\begin{array}{l}\text { Radiometer } \\
\text { Reproducibility }\end{array}$ & $E$ & Normal & 1 \\
\hline $\begin{array}{l}\text { Height Caliper } \\
\text { reproducibility }\end{array}$ & $\delta_{m i h .}$ & Normal & 1 \\
\hline Leakage Beam & $\delta_{S t r .}$ & Normal & 1 \\
\hline Color Temperature & $T_{d}$ & Normal & 0,7 \\
\hline Dark Measurement & $E_{0}$ & Normal & 1 \\
\hline
\end{tabular}

\subsubsection{Uncertainty of resolution, $\delta_{\text {res }}$}

$\mathrm{R}$ is the smallest measured value on the radiometer. When we calculate the digital radiometers uncertainty, we use equation (3) which is given below.

$$
\delta_{\text {res }}=\frac{R}{2 \sqrt{3}}
$$

When we calculate the analog radiometers uncertainty, we use equation (4) which is given below.

$$
\delta_{\text {res }}=\frac{R}{\sqrt{3}}
$$

\subsubsection{Uncertainty of repeatability, $E$}

The average of the measurements taken for each measurement point;

$$
\bar{x}=\frac{x_{1}+x_{2}+x_{3} \ldots x_{n}}{n}
$$

$\mathrm{n}$ : Number of Measurement

The experimental standard deviation for each measurement is calculated by the following formula; 


$$
S(x)=\sqrt{\frac{1}{n-1} \sum_{i=1}^{n}\left(\bar{x}-x_{i}\right)^{2}}
$$

The experimental standard deviation of the mean is calculated from the following formula;

$$
E=\frac{S(x)}{\sqrt{n}}
$$

E takes into account the largest of the standard deviation. When the results are evaluated, the standard deviation of the mean obtained is taken as the largest

\section{Conclusion}

Comparison Tests at Roketsan, The accuracy of the test, test and calibration activities and the accuracy of the results produced on the final product have a great impact on the final product. Periodic participation in ILC Tests, one of the most important guidelines for achieving the targeted reliability and accuracy level and planning continuous improvements, is provided in many areas. At this point, Roketsan Laboratories guarantee the accuracy and reliability of the activities carried out with the successful results obtained in performance evaluation.

In addition to ILC tests, Gage R \& R (Reproducibility and Reproducibility Competence Analysis) and MSA (Measurement System Analyze) studies, which play an important role in the analysis and development of laboratories and measurement systems, are being carried out intensively in our company. The results obtained through these internal applications are statistically analyzed and the actions are taken to improve the equipment, personnel, methodology and all external factors affecting the result.

As the Calibration Unit, we try to participate in interlaboratory comparison / proficiency tests not only for the scope of accreditation but also for other scopes to see laboratory performance. In this way, the continuity of laboratory competence is monitored.

Kemal Berk SÖNMEZ acknowledges the support of Roketsan Missiles Industries Inc.

\section{References}

1. International Organization for Standardization, ISO/IEC 17025 - General requirements for the competence of testing and calibration laboratories, (2017).

2. European Accreditation, EA-4/18 Guidance on the level and frequency of proficiency testing participation, (2010).

3. International Organization for Standardization, ISO/IEC $10012: 2003$, Measurement management systems - Requirements for measurement processes and measuring equipment (2015).

4. International Organization for Standardization, ISO/IEC 17043 :2010, Conformity assessment - General requirements for proficiency testing, (2010).

5. R. Hogan, The Ultimate Guide to Proficiency Testing for ISO/IEC 17025 Accreditation, (2019).

6. International Organization for Standardization, ISO/IEC 13528:2015, Statistical methods for use in proficiency testing by interlaboratory comparison, (2015)

7. C. Parr, Journal of Research of the National Institute of Standards and Technology, The Candela and Photometric and Radiometric Measurements, 106, Number 1, (2001) 